\title{
EARSHOT: A minimal neural network model of incremental human speech recognition
}

\author{
James S. Magnuson ${ }^{1,2}$, Heejo You ${ }^{1,2}$, Hosung Nam ${ }^{3,4}$, \\ Paul D. Allopenna ${ }^{1,2}$, Kevin Brown ${ }^{5}$, Monty Escabi ${ }^{1,2,6,7}$, Rachel M. Theodore ${ }^{1,8}$, \\ Sahil Luthra ${ }^{1,2}$, Monica $\mathrm{Li}^{1,2,4}$, Jay Rueckl ${ }^{1,2,4}$
}

\begin{abstract}
${ }^{1}$ Connecticut Institute for the Brain and Cognitive Sciences, University of Connecticut; ${ }^{2}$ Psychological Sciences, University of Connecticut; ${ }^{3}$ Korea University; ${ }^{4}$ Haskins Laboratories; ${ }^{5}$ Departments of Pharmaceutical Sciences and Chemical, Biological, and Environmental Engineering, Oregon State University; ${ }^{6}$ Electrical and Computer Engineering, University of Connecticut; ${ }^{7}$ Biomedical Engineering, University of Connecticut; ${ }^{8}$ Speech, Language, and Hearing Sciences, University of Connecticut
\end{abstract}

\begin{abstract}
The phenomenon of phonetic constancy is one of the greatest unsolved challenges in science. When humans hear speech, we readily perceive words and constituent phonemes (consonants and vowels) despite an absence of invariant acoustic cues. Instead, there is a many-to-many mapping from acoustics to percepts. Depending on phonetic context, speaking rate, and the dialect of the talker, one acoustic pattern can map to multiple phonemes, while multiple acoustic patterns can map to the same phoneme. Even as computer systems based on deep learning neural networks provide increasingly robust automatic speech recognition, a scientific understanding of human speech recognition has improved only incrementally over the past several decades, in part because most computational models that guide theories do not operate on real speech or learn, in order to keep them simple enough to understand. We report that a simple (two-layer) neural network that borrows one computational element from automatic speech recognition (long short-term memory nodes) can learn to map speech inputs to semantics with high accuracy and moderate generalization to new words and talkers while simulating the fine-grained time course of lexical activation and phonological competition observed in human listeners. Even though no explicit phonetic labeling is provided during training, analyses of hidden (modelinternal) node states over time reveal an emergent phonological code that resembles responses to speech in human cortex. This approach provides a unified framework for explaining the development, processes, and neural basis for human speech recognition, while allowing direct comparisons of human and model performance using real speech.
\end{abstract}

\section{INTRODUCTION}

Phonetic constancy in human speech recognition poses a significant theoretical challenge for the cognitive and neural sciences. Despite a lack of invariance (a many-to-many mapping between speech acoustics and linguistic percepts such as consonants, vowels, syllables, and words), listeners achieve phonetic constancy, perceiving a speaker's intended message with ease. Myriad factors can change the mapping from acoustics to percepts. The acoustic patterns specifying different phonemes overlap in time (Liberman, Cooper, Shankweiler, \& Studdert-Kennedy, 1967), with few boundaries between phonemes or words (Cole, \& Jakimik, 1980), and shift with factors such as speaking rate (Miller \& Baer, 1983), talker characteristics (Joos, 1948; Peterson \& Barney, 1952), phonetic context (Liberman et al., 1967), coarticulation (Liberman,
Delattre, \& Cooper, 1952), and novelty (Fowler \& Housum, 1987). Although similar problems exist in other domains (e.g., robust visual object recognition over variation in size, rotation, and illumination; DiCarlo \& Cox, 2007), the temporal nature of speech exacerbates the challenge.

If you use automatic speech recognition on your smartphone, you might assume that this challenge has been solved. Deeplearning neural network models underlying automatic speech recognition (Hinton, Deng, Yu, Dahl, Mohamed, Jaitly, Senior, Vanhoucke, Nguyen, Sainath, \& Kingsbury, 2012) provide amazingly robust real-world computer speech recognition but offer little guidance to theories of human speech recognition. Deep networks have many layers of richly connected nodes and typically require carefully engineered, biologically implausible training regimens. Scientists have used less complex deep networks to investigate mechanisms that might support audition and speech. For example, hidden units of a 5-layer network trained explicitly on phoneme recognition (Nagamine, Seltzer, \& Mesgarani, 2015) exhibited phonetically organized responses similar to those observed in human superior temporal gyrus (Mesgarani, Cheung, Johnson, \& Chang, 2014). Another deep network (Kell, Yamins, Shook, Norma-Haignere, \& McDermott, 2018) achieved human-like accuracy on one speech task (identifying the word at the center of a two second speech sample) and one music task (genre identification), with many layers and a complex training regimen. The network had 7 initial layers shared for the two tasks, and then branched into 5 separate layers for each task. The model was better at predicting human fMRI responses to natural sounds than was a standard model of auditory cortex based on spectrotemporal filters. The authors suggested that deep learning may be the only computational approach capable of human-like performance in perceptual domains. However, this approach has three important limitations: (i) unpacking emergent mechanisms in deep networks and linking them to theories of human capacities is a formidable challenge; (ii) many deep learning approaches to auditory processing do not take overtime input (instead, speech is input like an image, as though the entire sound occurred simultaneously); and, (iii) crucially, these models have not been applied to the complex time course of lexical activation and competition (a primary focus of cognitive theories).

Simpler models have guided theories of the time course of human speech recognition but have two different limitations. First, they do not use real speech as input; since the 1970s, most research on spoken word recognition has adopted the 
simplifying assumption that speech perception provides something like phonemic input to processes for word recognition. Neurally-inspired modeling of speech perception continued (Grossberg, Boardman, \& Cohen, 1997), but in small-inventory models rather than large vocabulary, signal-toword models. Recent attempts at linking automatic speech recognition approaches to cognitive models (Scharenborg, O., 2010; Scharenborg, Norris, ten Bosch, \& McQueen, 2005) have generated interesting insights, but with low accuracy and quite limited empirical coverage. Second, they set aside the problem of learning, using fixed parameters in neural network (McClelland \& Elman, 1986) and Bayesian approaches (Norris \& McQueen, 2008). Nonetheless, these models simulate the time course of lexical activation and phonological competition (Allopenna, Magnuson, \& Tanenhaus, 1998), and have significantly advanced an understanding of human speech recognition dynamics. The persistence of these "temporary" simplifying assumptions decades later reflects the tension between computational adequacy (maximizing task realism and performance) and psychological adequacy (capturing key details of human behavior, while providing an understandable account of how the model works; McClelland \& Elman, 1986). A model with high computational adequacy but opaque mechanisms offers little guidance to theories that seek to explain mechanisms supporting human speech recognition at a fine grain.

We would expand this set of adequacies. We divide psychological adequacy into three parts. Behavioral adequacy is the ability of a model to simulate key details of human performance. Developmental adequacy is firstly the ability of a model to learn and secondly the degree to which learning by the model is relatable to trajectories in human development. Explanatory adequacy is the degree to which the mechanisms of the model are analyzable and understandable; a model could have high adequacy in every other domain, but its utility in guiding theories of human capabilities will be limited if the mechanisms implemented in the model are not well understood. We would also complement computational and psychological adequacy with neural adequacy: the ability of the model to relate to knowledge or theories of the neurobiological mechanisms underlying the modeled capacities.

With this framework in mind, our goal was to develop a minimal (and thus more readily analyzable and understandable) cognitive model of human speech recognition that could learn to map over-time speech to semantics, without explicit phonetic training. By emphasizing simplicity, such a model could reveal representations that emerge in a simple learning system, providing hypotheses for cognitive and neural mechanisms supporting human speech recognition. Through explorations of dozens of networks borrowing a variety of elements from automatic speech recognition (see Methods), we achieved human-like performance with a shallow network that used long short-term memory (LSTM) nodes (Hochreiter \& Schidhuber, 1997). LSTM nodes add three internal gates and a memory cell that allow nodes to develop sensitivity to information over long time scales, mitigating the vanishing gradient problem (Hochreiter, Bengio, Frasconi, \& Schmidhuber, 2001): recurrent networks (with connections between nodes within a layer or from a superior to an inferior layer) can theoretically become sensitive to dependencies over long time spans, but in practice, context degrades at each time step, severely limiting

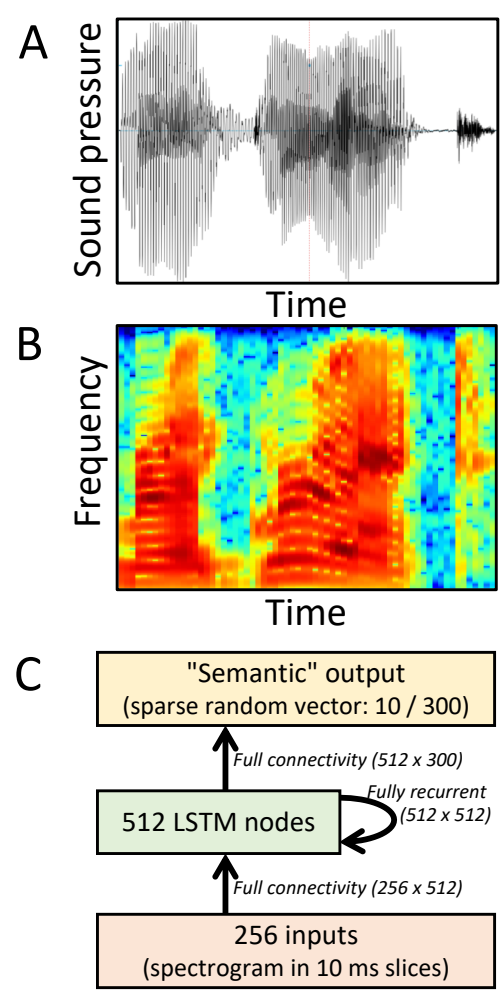

Fig. 1. Model input and structure. (A) Audio files are converted to spectrograms (B), with 256 channels (rows) in $10 \mathrm{~ms}$ steps (columns). Color indicates amplitude (blue-red indicates lowhigh). (C). The model is a standard recurrent network, except "long short-term memory" nodes are used in the hidden layer, allowing it to become sensitive to multiple temporal grains.

the span of learnable dependencies. As we shall see in the next section, LSTM nodes allow a shallow network to learn to map speech to approximations of semantic representations.

\section{METHODS}

Network architecture. We constructed a network (Fig. 1) dubbed EARSHOT, for Emulation of Auditory Recognition of Speech by Humans Over Time, emphasizing key aims of working with real speech and emulating the time course of human speech recognition. The model has 256 spectrographic inputs, 512 LSTM hidden nodes, and 300 pseudo-semantic outputs (random sparse vectors; a common simplification (Laszlo \& Plaut, 2012) given the largely arbitrary mapping from sound to meaning). There are feedforward connections between layers, and the hidden layer is fully recurrent (each node has a connection to every other). Connections were trained using backpropagation through time (Werbos, 1988). The training target at each time step was the semantic vector corresponding to the current word. We trained several models on 1,000 words produced by 10 talkers (see Materials). The maximum performance was approximately $90 \%$, which was achieved with a model with 512 hidden units. We tested models with as many as 768 hidden units, but accuracy did not improve.

Materials. A total of 1000 words with length 1-8 phonemes (mean: 5.5) were selected pseudo-randomly from a list of uninflected English words, with the constraint that each phoneme occur at least 10 times in the list of selected words. 
Pronunciations of all 1000 words were then generated from 10 talkers included in the Apple text-to-speech application, say (five females [Agnes, Kathy, Princess, Vicki, Victoria] and five males [Alex, Bruce, Fred, Junior, Ralph]). The sound files were $659 \mathrm{~ms}$ in duration on average (ranging from 289 to $1121 \mathrm{~ms}$ ). For analysis purposes, we created 360 consonant-vowel and 360 vowel-consonant syllable pronunciations for each talker. This total follows from using an inventory of 24 consonants and 15 vowels. Each sound file was converted to a spectrogram with 256 channels in $10 \mathrm{~ms}$ steps, and a frequency range of 0-3600 Hz. For each word, a unique semantic target pattern was created. As mentioned above, these were random sparse vectors, with 10 of 300 elements set to 1 , and all others set to 0 .

Training method. We created 10 different models that differed only in terms of their training items. For each model, a different talker was entirely excluded from training. For each of the nine included talkers, 100 different, randomly-selected words were excluded from training. Thus, the training set for each model consisted of 8,100 input-output patterns (900 words x 9 talkers). Training was organized as epochs. Each epoch included one presentation of each of the 8100 training items in random order with no pause or other indication of word boundaries except that the training target pattern changed (the target pattern was always the semantic vector for the current word). To increase learning speed and model performance, three techniques were used (Vaswani, Shazeer, Parmar, Uszkoreit, Jones, Gomez, Kaiser, \& Polosukhin, 2017): minibatch gradient descent, Noam decay, and Adam optimizing. The 8100 words were divided into 5 mini-batches (4 x 2000, 1 x 100). A baseline learning rate of 0.002 was applied adaptively using the Adam optimization method and Noam decay, as in the following equation.

$L R=0.002 \times 4000^{0.5} \times \min \left(E \times 5 \times 4000^{-1.5},(E \times 5)^{-0.5}\right)$

In this equation, $L R$ and $E$ denote learning rate and epoch, respectively. The hyper parameters $\beta_{1}, \beta_{2}$, and $\varepsilon$ of Adam optimizing were $0.9,0.999$, and $1 \mathrm{e}-08$, respectively.

Testing. To quantify the distance of the output vector at each time step to each word in the 1000-word lexicon, we computed the cosine similarity of the output vector to all 1000 semantic vectors.

Cosine similarity $=\left(\sum_{i=1}^{n} T_{i} \times O_{i}\right) /\left(\sqrt{\sum_{i=1}^{n} T_{i}^{2}} \times \sqrt{\sum_{i=1}^{n} O_{i}^{2}}\right)$

In this equation, $T, O$, and $n$ indicate the target, output, and vector length, respectively. $O$ indicates the output vector at one time step.

At the end of every 1000 epochs, each model was tested with all 10,000 words (including excluded words and excluded talkers). Accuracy was operationalized based on a 2-parameter threshold: the output vector's cosine similarity to the target had to exceed any other item's cosine similarity to the target by a minimum of 0.05 for at least $100 \mathrm{~ms}$, and subsequently, no item could exceed the target's cosine similarity to the target before word offset.

Simulation method (training and testing). The simulation process was as follows. First, each spectrogram $I$ was applied to the input layer at each time step. Hidden activation $H$ of each time step was derived through input $I$. The following formulas (26) were used for calculating $H$.

$$
\begin{aligned}
& i_{t}=\sigma\left(I_{t} W_{I i}+H_{t-1} W_{H i}+c_{t-1} W_{c i}+b_{i}\right) \\
& f_{t}=\sigma\left(I_{t} W_{I f}+H_{t-1} W_{H f}+c_{t-1} W_{c f}+b_{f}\right) \\
& c_{t}=f_{t} c_{t-1}+i_{t} \times \tanh \left(I_{t} W_{I c}+H_{t-1} W_{H c}+b_{c}\right) \\
& o_{t}=\sigma\left(I_{t} W_{I o}+H_{t-1} W_{H o}+c_{t} W_{c o}+b_{o}\right) \\
& H_{t}=\tanh \left(o_{t} \times \tanh \left(c_{t}\right)\right.
\end{aligned}
$$

In the equations above, $i, f$, and $o$ denote input, forget, and output LSTM gates, respectively. $c$ is the LSTM cell memory, and $W$ is the weight that connects two subscripted nodes. $b$ is the bias of the subscripted node. $\sigma$ and tanh are activation functions. Based on the derived $H$, the semantic output activation $O$ was derived using the following equation.

$$
O_{t}=\sigma\left(H_{t} W_{H O}+b_{o}\right)
$$

$O$ was derived for all time steps, and backpropagation was also performed for $O$ at all time steps.

Additional details. See Appendix 1 for additional details.

\section{RESULTS}

Models achieved high accuracy after 8000 training epochs (Fig. 2): $88 \%$ for trained-on items, $67 \%$ for excluded words from training talkers, and 33\% for excluded talkers (range: $4 \%$ to $78 \%$ ). Generalization was poor for some talkers, but human listeners can learn to adapt to novel talkers. When we resumed training with all items from all talkers included, performance improved rapidly (to $89 \%$ and $86 \%$ for excluded words and talkers, and a boost to $93 \%$ for previously trained items). Accuracy details by talker are presented in Appendix 2.

While high accuracy is a prerequisite for a valid model, a greater challenge is simulating the time course of human speech recognition (Allopenna et al., 1998). The time course of human speech recognition is a crucial explanatory target in speech science, but previous deep learning models of speech (Kell et al., 2018; Nagamine et al., 2015) have not been applied to time course. Our minimal model exhibits the correct qualitative pattern for phonological competition (Fig. 2). It is not necessarily the case that any model that can map speech inputs to semantic outputs would exhibit human-like time course; we discuss examples of high-accuracy models with time course behavior that differs starkly from human performance in the Methods.

The next challenge is determining how the model works, with the aim of guiding theories of human speech recognition at both cognitive and neural levels; the representations and transformations that emerge in this simple learning system could provide hypotheses for neural mechanisms supporting human speech recognition. To begin, we adapted two "sensitivity indices" (SIs) used with human electrocorticography data (Mesgarani et al., 2014). The Phonetic Sensitivity Index (PSI) for a hidden unit-phoneme pair is the count of phonemes that evoke a substantially weaker response in that unit compared to the target phoneme. For example, given 39 phonemes, if a hidden unit responds more strongly to /p/ than any other phoneme, its PSI for /p/ would be 38 (maximum). The Featural Sensitivity Index (FSI) does the same for features shared by classes of phonemes (e.g., nasal, labial, voiced). The SI approach allows us to ask whether phonetic structure emerges as the model learns to map speech to semantics, despite not being given explicit information about phonetic features or phonemes.

We used hierarchical clustering to sort hidden units based on SIs (Fig. 3). Approximately 50\% of nodes exhibit structured responses in the SI time window (in the human 

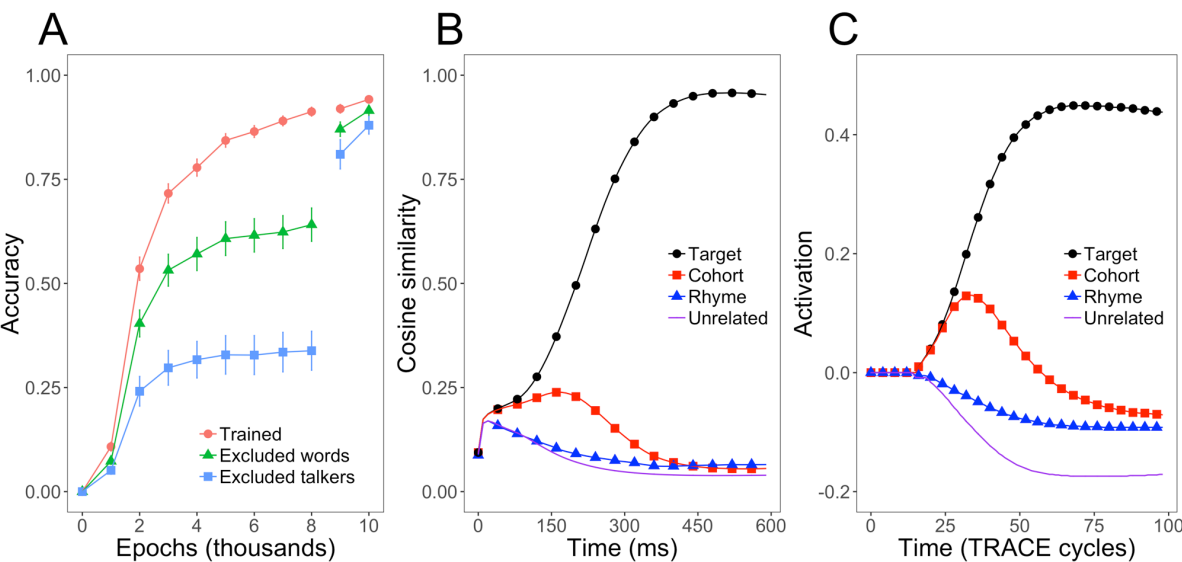

Fig. 2. Model performance. (A) Accuracy by epoch averaged over ten models. When training resumed with all items (epochs $8001-10,000$ ), high accuracy was achieved quickly for all talkers. (B) The time course of competition for accurate trials, for 2 criterial competitor types. For a target (e.g., cat), "Cohort" represents mean cosine similarity for words overlapping in the first two phonemes (can, castle). "Rhymes" rhyme with the target (bat, sat). "Unrelated" is the average for all words phonologically dissimilar from the target. This pattern closely follows human performance (Allopenna et al., 1998). (C) For comparison, we conducted simulations with the TRACE model, with its standard 212-word lexicon, 14-phoneme inventory, and idealized "pseudo-spectral" inputs. Crucially, the same rank ordering and similar timing is observed in both of competitor types is similar in the two models.

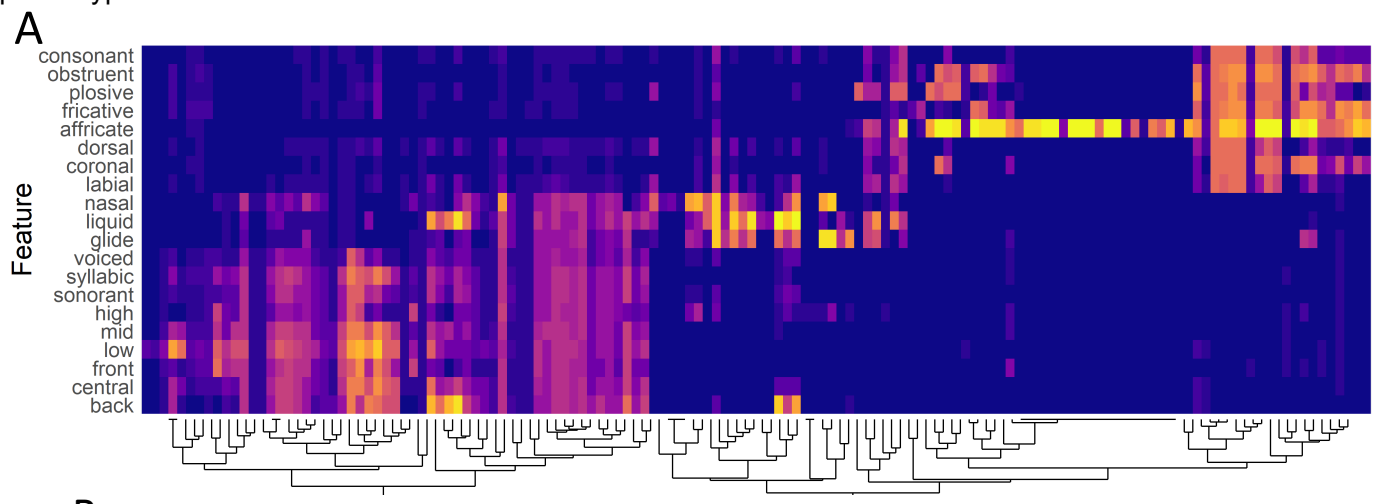

B

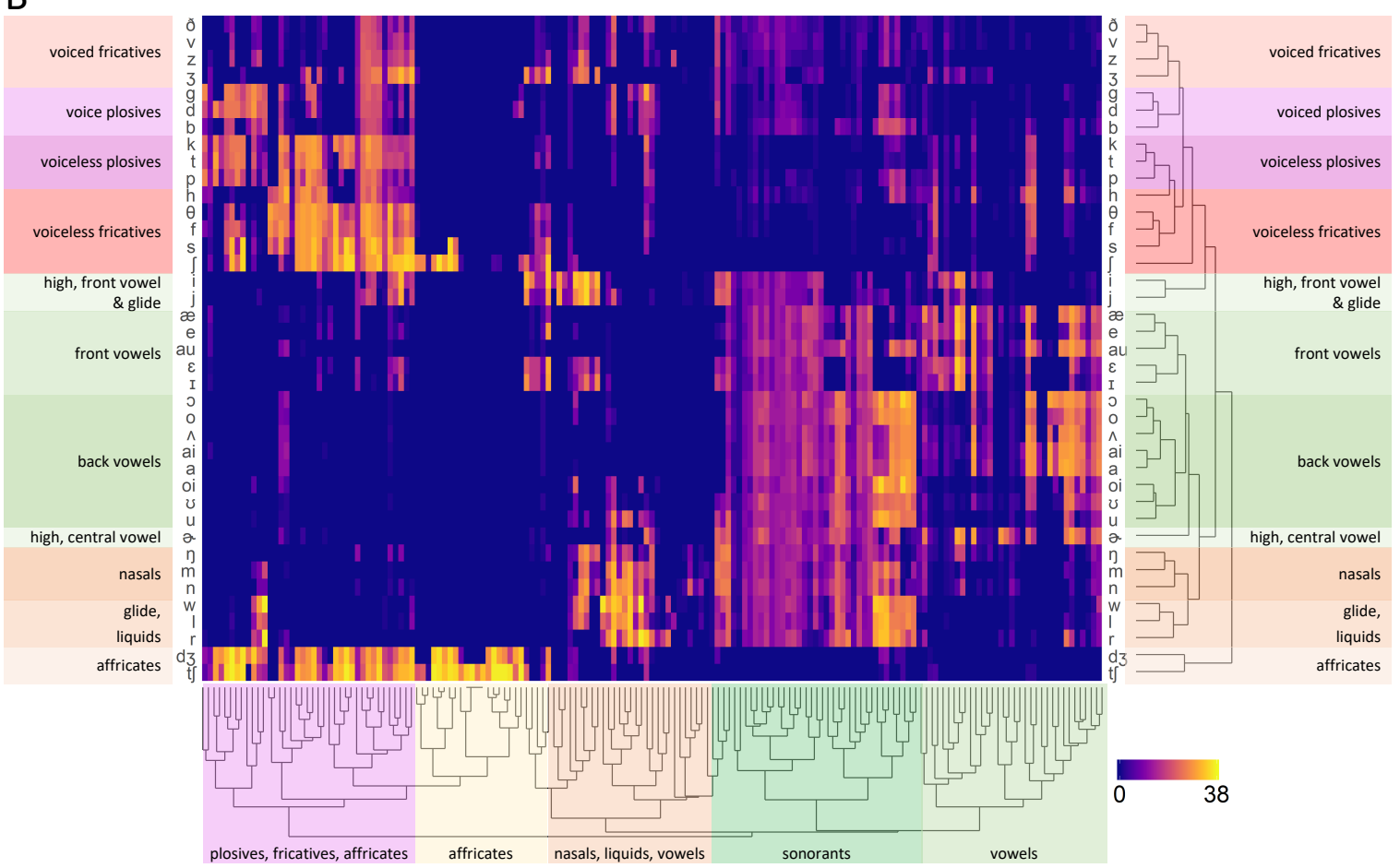

Fig. 3. Phonetic sensitivity revealed by hierarchical clustering. (A) Featural Sensitivity Index (FSI) based on hidden unit ( $\mathrm{x}$-axis) responses to phonetic features; for every hidden unit-feature pair, FSI was incremented for every feature to which the hidden unit responded substantially more weakly (yellow indicates high selectivity, with maximum FSI of 19, given 20 features). Vowel features pattern together (from high to back). $187 \mathrm{HUs}$ with strongly selective responses are included. (B) Phonetic Sensitivity Index (PSI). High PSI indicates selective responses to specific phonemes. Maximum score is 38 , given 39 phonemes. 246 HUs showing selective responses are included. 


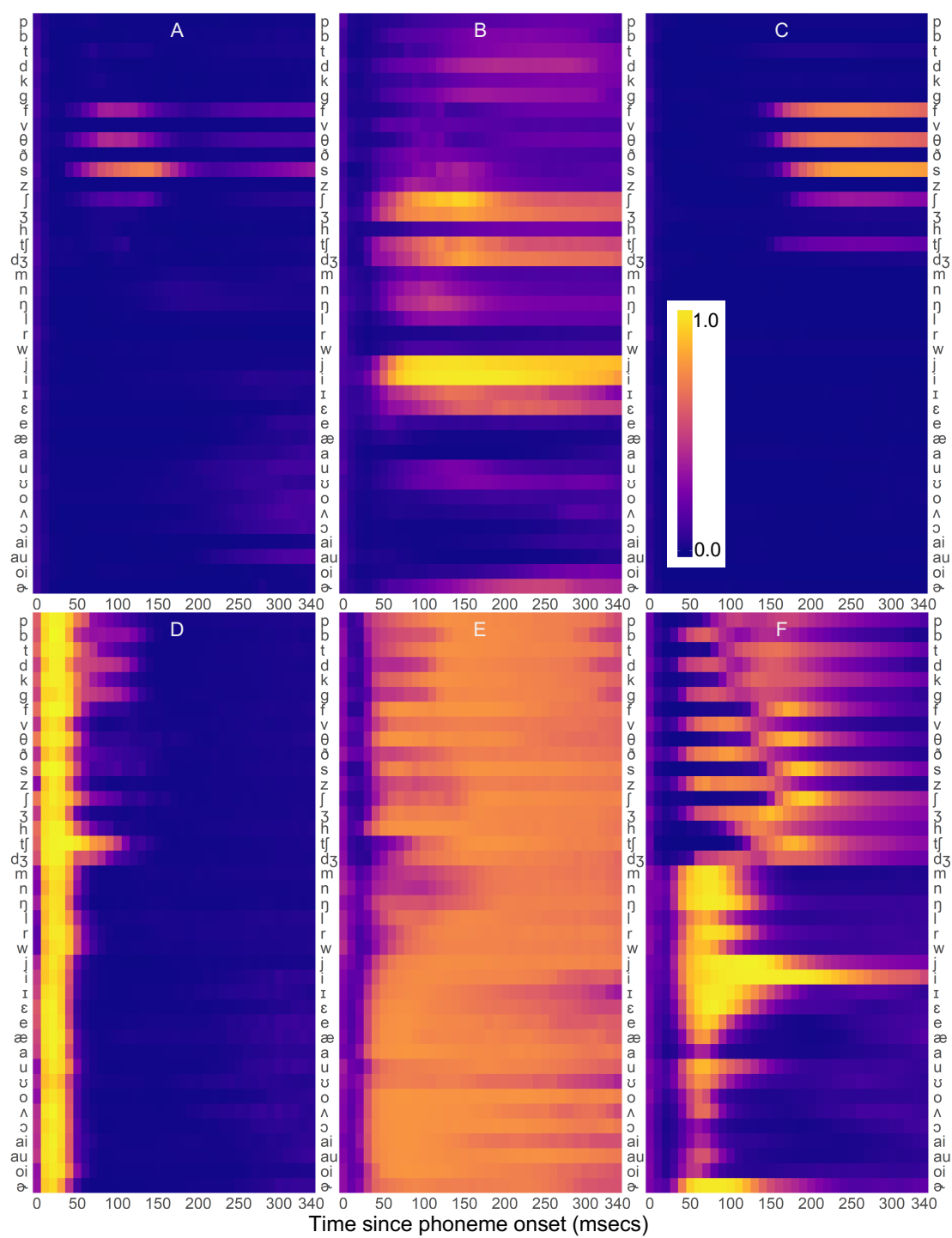

Fig. 4. Hidden unit response profiles. Over-time response profiles of example hidden units for each phoneme (y-axis). (A) Time locked, discrete responses (5\% of units). (B) Time locked, sustained responses (20\%). (C) Delayed responses (35\%). (D) Early onset responses (4\%). (E) Post-onset inactivation (3\%). (F) Complex responses (29\% of HUs). An additional $4 \%$ are largely non-responsive.

(Mesgarani et al., 2014), approximately $20 \%$ of electrodes met criteria for inclusion in SI analyses). The FSIs and PSIs are remarkably similar to those derived from electrodes placed in human superior temporal gyrus (Mesgarani et al., 2014), with selective responses to features and phonetically similar phonemes.

The sensitivity indices reveal an internal phonetic code that emerges in the model. However, hidden units have more complex dynamics than are revealed by the SIs (Fig. 4). Some develop strong, onset-locked responses, while others develop responses that include significant delays, and/or sustained responses. These response profiles suggest novel hypotheses for human cortical responses that could be explored in electrocortiographic recordings. The mapping from hidden states over time to semantic outputs likely depends both on intuitive profiles like the time-locked responses assumed by the SI analyses and on complex over-time patterns of combinations of those and other profiles. Additional details of hidden unit profiles and responses are presented in the Appendix 2.

\section{DISCUSSION}

More than 60 years after discovery of the lack of invariance problem - the absence of invariant cues to speech sounds (e.g., Joos, 1948; Liberman et al., 1952; Peterson \& Barney, 1952) speech science offers limited explanations of how humans achieve phonetic constancy despite the many-to-many mapping between acoustics and percepts. We argue that a significant impediment to understanding is that computational models of human speech recognition since the 1970s have typically embraced what was meant to be a temporary simplifying assumption that divides labor, with speech perception research focused on phonetic constancy, and modeling focused on word recognition (McClelland \& Elman, 1986; Norrs \& McQueen, 2008). The latter depends on the simplifying assumption that processes of speech perception output idealized, abstract elements such as phonetic features (McClelland \& Elman, 1986), phonemes (Hannagan, Magnuson \& Grainger, 2013; You \& Magnuson, 2018), or human phoneme confusion 
probabilities (Norris \& McQuen, 2008) rather than real speech. Simplifying assumptions like this can ultimately complicate problems (25), as the details they sidestep may contain constraints essential to the mechanisms underlying human performance.

A primary obstacle to developing models that take real speech as input is concerns about complexity. As McClelland and Elman argued (McClelland \& Elman, 1986), a computational model aimed at guiding a psychological theory must prioritize psychological adequacy over computational adequacy. That is, such a model must favor simplicity and understandability (what we referred to as explanatory adequacy above) over full, end-to-end modeling when the latter results in a model too complex to understand. The EARSHOT model brings this tension into sharp relief. We demonstrated that borrowing one element from automatic speech recognition long short-term memory (LSTM) nodes (Hochreiter \& Schmidhuber, 1997) - is sufficient to allow a shallow recurrent network to learn to map from speech to pseudo-semantics, while also demonstrating a time course of apparent lexical activation and competition (Fig. 2) that resembles that observed in human subjects and current gold-standard models of human speech recognition (Allopenna et al., 1998). As a learning model, EARSHOT has potential developmental adequacy as well (although we have not yet attempted to make the training of the model realistic or to link its development to human developmental trajectories).

However, EARSHOT's explanatory adequacy is not clear, since how the model succeeds in learning to map speech to semantics is not immediately apparent. EARSHOT has substantially greater computational adequacy than a model like TRACE (McClelland \& Elman, 1986), but it is also more difficult to understand. We demonstrated that we can begin to understand how EARSHOT learns to map speech to semantics by using techniques from human electrocorticography (Mesgarani et al., 2014) to track responses of hidden units to specific phonetic features and phonemes (Figs. 3 and 4). EARSHOT's emergent sensitivity to phonetic structure, despite receiving no explicit phonetic training, provides some preliminary clues as to how such a simple learning system could achieve a speech-to-semantics mapping. Of course, fully understanding how the model works will require analyses beyond the phonetic structure apparent from the featural and phonetic sensitivity indices. First, it is apparent from the variation in hidden unit response profiles (Fig. 4) that the phonetic responses of the hidden units are substantially more complex than the sensitivity indices suggest. We expect that complex population responses are essential to how the model transforms spectral slices to semantics (rather than a system where individual units function as simple detectors for specific phonemes). Second, a full understanding of the model will also require unpacking the transformation from hidden unit states to semantic outputs. However, even the preliminary similarity of EARSHOT's hidden unit responses to human electrocortiographic data suggests that such a model holds promise for addressing neural adequacy. Indeed, an intriguing possibility is that the variations in response profiles observed could generate hypotheses for human cortical responses to speech.

In conclusion, by borrowing minimal elements from automatic speech recognition, EARSHOT opens new territory to computational exploration of human speech recognition. The fundamental challenges of the lack-of-invariance problem, which has been outside the scope of cognitive models of human speech recognition for decades, are now addressable. These include variation in talker characteristics, speaking rate, and acoustic context, and integration of theories of development and processing. Simulations can be conducted with the same materials presented to human listeners, instead of idealized, abstract analogs of those materials. Finally, the fact that the distributed phonological code that emerges as the model learns to map speech to semantics resembles responses observed in human cortex (Mesgarani et al., 2014) demonstrates the promise of this approach as a testbed for theories of neurobiological mechanisms that may support human speech recognition.

\section{REFERENCES}

Allopenna, P.D., Magnuson, J.S., \& Tanenhaus, M.K. (1998). Tracking the time course of spoken word recognition using eye movements: Evidence for continuous mapping models. Journal of Memory and Language 38, 419-439 (1998).

Cole, R.A., \& Jakimik, J. (1980). A model of speech perception. In R.A. Cole (Ed.), Perception and production of fluent speech (Erlbaum), pp 133-163.

DiCarlo, J.J., \& Cox, D.D. (2007). Untangling invariant object recognition. Trends in Cognitive Sciences 11, 333-341.

Elman, J.L. (1990). Finding structure in time. Cognitive Science 14, 179-211.

Fowler, C.A., \& Housum, J. (1987). Talkers' signaling of "new" and "old" words in speech and listeners' perception and use of the distinction. Journal of Memory and Language 26, 489-504.

Grossberg, S., Boardman, I., \& Cohen, M. (1997). Neural dynamics of variable-rate speech categorization. Journal of Experimental Psychology: Human Perception and Performance 23, 418-503.

Hannagan, T., Magnuson, J.S., \& Grainger, J. (2013). Spoken word recognition without a TRACE. Frontiers in Psychology, 4:563. doi:10.3389/fpsyg.2013.00563.

Hinton, G., Deng, L., Yu, D., Dahl, G., Mohamed, A., Jaitly, N., Senior, A., Vanhoucke, V., Nguyen, P., Sainath, T., \& Kingsbury, B. (2012). Deep neural networks for acoustic modeling in speech recognition: the shared views of four research groups. IEEE Signal Processing Magazine 29, 82-97.

Hochreiter, S., \& Schmidhuber, J. (1997). Long short-term memory. Neural Computation 9, 1735-1780.

Hochreiter, S., Bengio, Y., Frasconi, P., \& Schmidhuber, J. (2001). Gradient flow in recurrent nets: the difficulty of learning long-term dependencies. A Field Guide to Dynamical Recurrent Neural Networks, eds Kremer SC, Kolen JF (IEEE Press), pp. 237-374.

Joos, M. (1948) Acoustic phonetics. Baltimore: Linguistic Society of America.

Kell, A.J.E., Yamins, D.L.K., Shook, E.N., Norma-Haignere, S.V., \& McDermott, J.H. (2018). A task-optimized neural network replicates human auditory behavior, predicts brain responses, and reveals a cortical processing hierarchy. Neuron 98, 630-644.

Laszlo, S., \& Plaut, D.C. (2012). A neurally plausible parallel distributed processing model of event-related potential reading data. Brain and Language 120, 271-281. 
Liberman, A.M., Cooper, F.S., Shankweiler, D.P., \& Studdert-Kennedy, M. (1967). Perception of the speech code. Psychological Review, 74, 431-461.

Liberman, A.M., Delattre, P.C., \& Cooper, F.S. (1952). The role of selected stimulus variables in the perception of the unvoiced-stop consonants. American Journal of Psychology 65, 497-516.

Magnuson, J.S. (2008). Nondeterminism, pleiotropy, and single word reading: Theoretical and practical concerns. Single Word Reading, eds Grigorenko E., Naples E. (Erlbaum), pp. 377-404.

McClelland, J.L., \& Elman, J.L. (1986). The TRACE Model of Speech Perception. Cognitive Psychology 18, 1-86.

Mesgarani, N., Cheung, C., Johnson, K., \& Chang, E.F. (2014). Phonetic feature encoding in human superior temporal gyrus. Science 343, 1006-1010.

Miller, J.L., \& Baer, T. (1983). Some effects of speaking rate on the production of $/ \mathrm{b} /$ and $/ \mathrm{w} /$. Journal of the Acoustical Society of America 73, 1751-1755.

Nagamine, T., Seltzer, M.L., \& Mesgarani, N. (2015). Exploring how deep neural networks form phonemic categories. Sixteenth Annual Conference of the International Speech Communication Association, Dresden, Germany, 19121916.

Norris, D., \& McQueen, J.M. (2008). Shortlist B: A Bayesian model of continuous speech recognition. Psychological Review 115, 357-395.

Peterson, G.E., \& Barney, H.L. (1952). Control methods used in a study of vowels. Journal of the Acoustical Society of America 24, 175-184.

Scharenborg, O. (2010). Modeling the use of durational information in human spoken-word recognition. Journal of the
Acoustical Society of America 127, 3758-3770.

Scharenborg, O., Norris, D., ten Bosch, L., \& McQueen, J.M. (2005). How should a speech recognizer work? Cognitive Science 29, 867-918.

Vaswani, A., Shazeer, N., Parmar, N., Uszkoreit, J., Jones, L., Gomez, A.N., Kaiser, L., \& Polosukhin, I. (2017) Attention is all you need. arXiv:1706.03762v5 [cs.CL].

Werbos, P.J. (1988). Generalization of backpropagation with application to a recurrent gas market model. Neural Networks 1, 339-356.

You, H., \& Magnuson, J.S. (2018). TISK 1.0: An easy-to-use Python implementation of the time-invariant string kernel model of spoken word recognition. Behavior Research Methods. doi:10.3758/s13428-017-1012-5.

\section{ACKNOWLEDGMENTS}

We thank Inge-Marie Eigsti, Blair Armstrong, Thomas Hannagan, and Ram Frost for comments. This work was supported by the following grants: NSF 1754284 (PI: JSM), NSF IGERT 1144399 (PI: JSM), NSF NRT 1747486 (PI: JSM), NICHD P01 HD0001994 (PI: JR), and NSF 1827591 (PI: RMT).

\section{AUTHOR CONTRIBUTIONS}

J.S.M., H.Y., and H.N. conceived of the initial project; all authors contributed to interpretation of results and development of the data analysis strategies, and to the writing of the manuscript; H.Y. implemented the model and analysis scripts; J.S.M. wrote the original draft of the manuscript. 


\section{APPENDIX 1: ADDITIONAL METHODOLOGICAL DETAILS}

\section{SENSITIVITY INDICES}

The phoneme sensitivity index (PSI) of hidden units was calculated as follows. First, we calculated the absolute value of each hidden unit's activation over time in response to all $\mathrm{CV}$ - and VC-diphones. Then, for each initial phoneme, we averaged each hidden unit's response to all diphones beginning with that segment, to derive the mean response of each hidden unit to each phoneme. We found that the modal maximal response period across all hidden units in response to all phonemes occurred from $0-60 \mathrm{~ms}$ after phoneme onset. We thus characterized the response of each hidden unit to each phoneme as the mean response to all diphones beginning with that phoneme over the $0-60 \mathrm{~ms}$ time period. Then for each phoneme-hidden unit pair, we calculated a PSI value as follows. Phoneme $(i)$-hidden unit $(j)$ pair $P_{i j}$ received 1 point for every phoneme to which hidden unit $j$ responded more weakly than it did to phoneme $i$ by a threshold (0.15). So for example, if the activation of hidden unit 207 in response to $/ \mathrm{p} /$ exceeded its response to $/ \mathrm{b} / \mathrm{by}$ 0.24 , the PSI for $P_{/ \mathrm{p} / 207}$ was incremented. The maximum PSI was 38 , which would indicate that the response of a hidden unit to a particular phoneme exceeded the threshold difference for all other phonemes.

In the study that motivated our use of the PSI (Mesgarani et al., 2014), the Wilcoxon rank sum test was used to compare electrode responses to phoneme pairs, with PSIs incremented when the difference was significant. However, we used a simple criterion in this study because very small differences easily reached significance. The threshold of 0.15 provided a level of sparsity similar to that reported in human sensitivity indices ${ }^{13}$.

To examine structured responses via the PSI, we used simple hierarchical clustering of each hidden unit's PSIs for all 39 phonemes (Fig. 4). Any hidden units that had PSIs of 0 for all phonemes were excluded.

The Featural Sensitivity Index (FSI) uses the same method as the PSI, but linked to features rather than phonemes. For example, for the FSI to "voiced", all diphones with a voiced segment in the first position were used. Our feature definitions are listed in Table S1.

\section{REPLICABILITY}

Replicability was confirmed by repeating the complete training of 10 models three times; only minor variations were observed between iterations.

\section{HARDWARE AND SOFTWARE}

Simulations were conducted on a Windows 10 workstation with an i7-6700k CPU, 64-gb of RAM, and a Titan-X (12gb) graphics card. Simulations were implemented using Python 3.6 and TensorFlow 1.7. Each model requires approximately 10 hours to train on this workstation. Upon request, we can supply an up-to-date Linux container with all necessary software and libraries for running our simulation code. However, conducting simulations will still require a high-performance workstation.

\section{ALTERNATIVE ARCHITECTURES}

In developing this model, we explored dozens of combinations of candidate architectures and inputs. All were limited to two layers (that is, inputs, hidden units, and outputs; the layer count is based on the number of layers after the input). For architectures, we varied three aspects of models: hidden unit size (which we typically varied from 100 to 1000 nodes before rejecting an architecture for accuracy below 90\%), hidden unit type (standard integrative nodes vs. LSTMs), and degree of recurrence (full recurrence, as in the model reported here, vs. single-step recurrence, as in simple recurrent networks; Elman, 1990). For inputs, we tried spectrograms at various resolutions, Mel Frequency Cepstral Coefficients (MFCCs), and cochleagrams.

Most combinations failed to achieve high accuracy. The only combinations that achieved greater than $90 \%$ accuracy were those reported here for EARSHOT and a similar model using low-dimensional MFCCs rather than spectrographic inputs. However, the latter failed to show realistic time course (see Fig. A1.1).

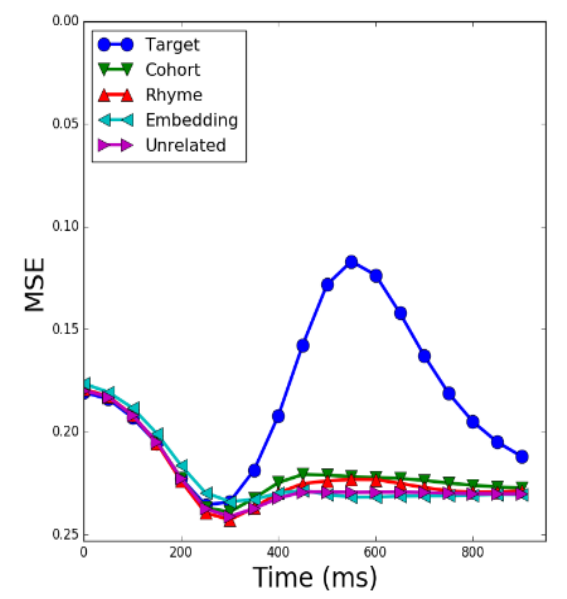

Fig. A1.1. Unrealistic time course in a high-accuracy model. This example illustrates that the correct time course does not necessarily emerge from any model with high accuracy. For this model, we used 13-element Mel Frequency Cepstral Coefficients (MFCCs, a common transformation used in ASR) as inputs to 500 LSTM nodes that mapped onto 300 semantic outputs. The model achieved $95 \%$ accuracy on a 200 -word lexicon produced by 10 talkers. Here, we tracked mean squared error (MSE) to each pattern (note that the MSE scale is reversed to facilitate comparison to Fig. 2 in the main text), and a radically unrealistic time course (compared to human behavior; see Fig. 2 in the main text) emerged. 
FEATURES

IPA consonant obstruent plosive fricative affricate dorsal coronal labial nasal liquid glide voiced syllabic sonorant high mid low front central back IPA

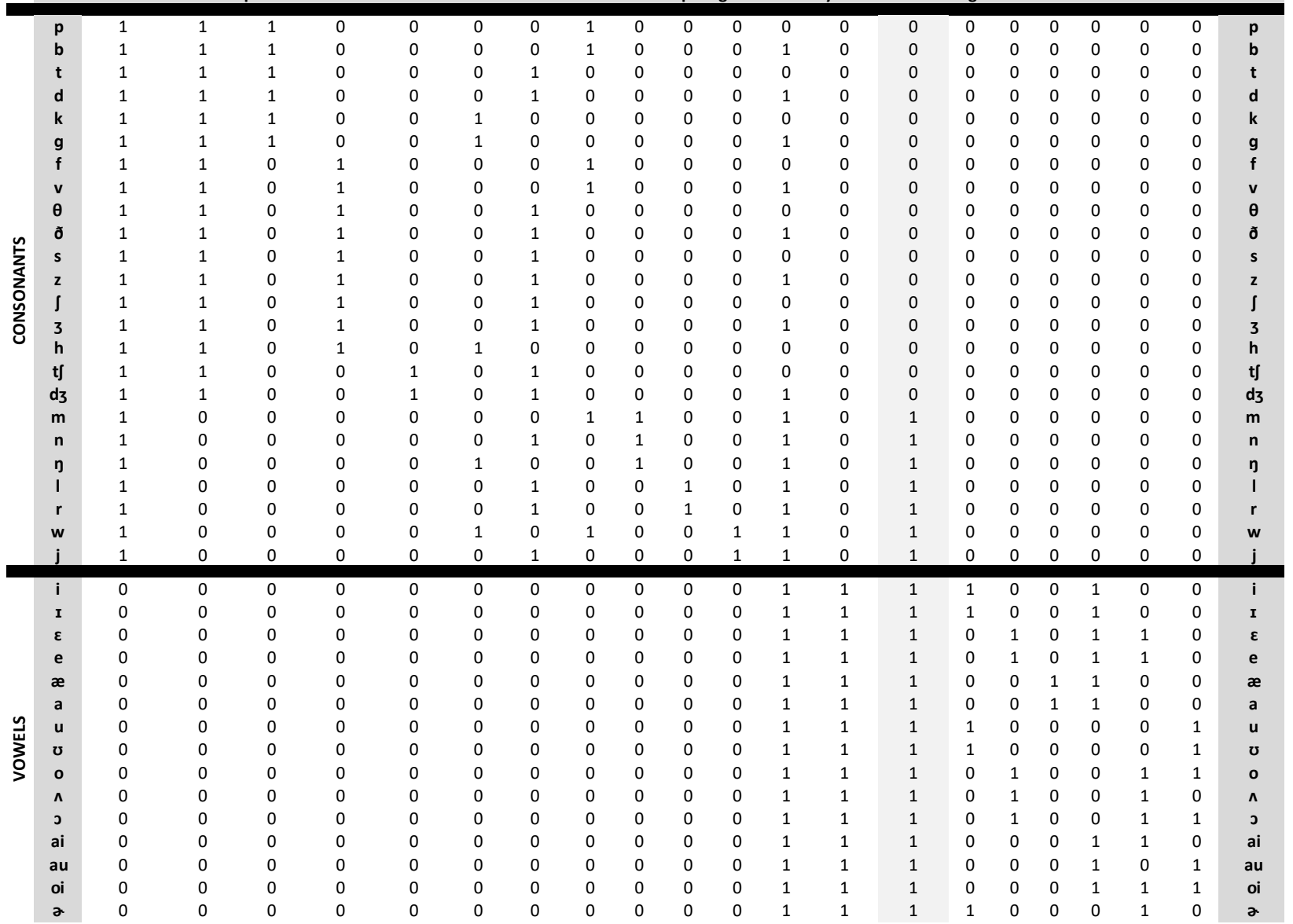

Table A1.1: Phoneme-feature correspondences. The thick horizontal border separates consonants and vowels. The feature 'sonorant' is highlighted as the one feature that includes consonants and vowels. 


\section{APPENDIX 2: ADDITIONAL RESULTS}
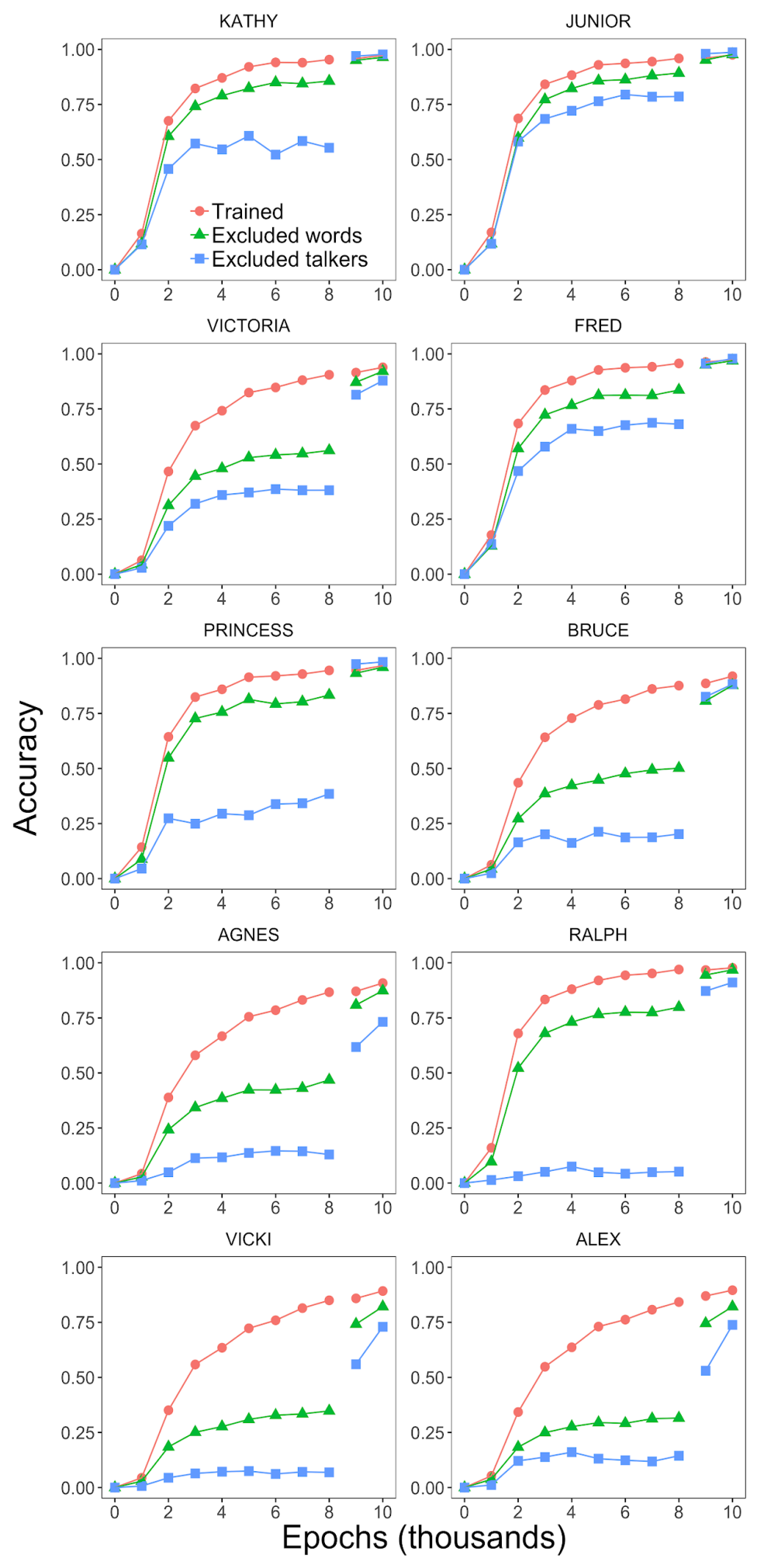

Fig. A2.1. Accuracy over epochs organized by talkers. In each panel, "Trained" indicates the performance on the listed talker (e.g., "KATHY") in the 9 simulations where it was included. "Excluded words" indicates performance on the listed talker's 100 excluded words in the 9 models that included that talker in training. The "Excluded talker" lines track performance on the listed talker when it was excluded. Training for each model was conducted for 8000 epochs with 100 words per training talker excluded and one talker excluded completely. For epochs $8001-10,000$, excluded items were introduced to the training set. As can be seen from the figure, even for talkers for which generalization was initially poor, training allowed rapid improvement. 


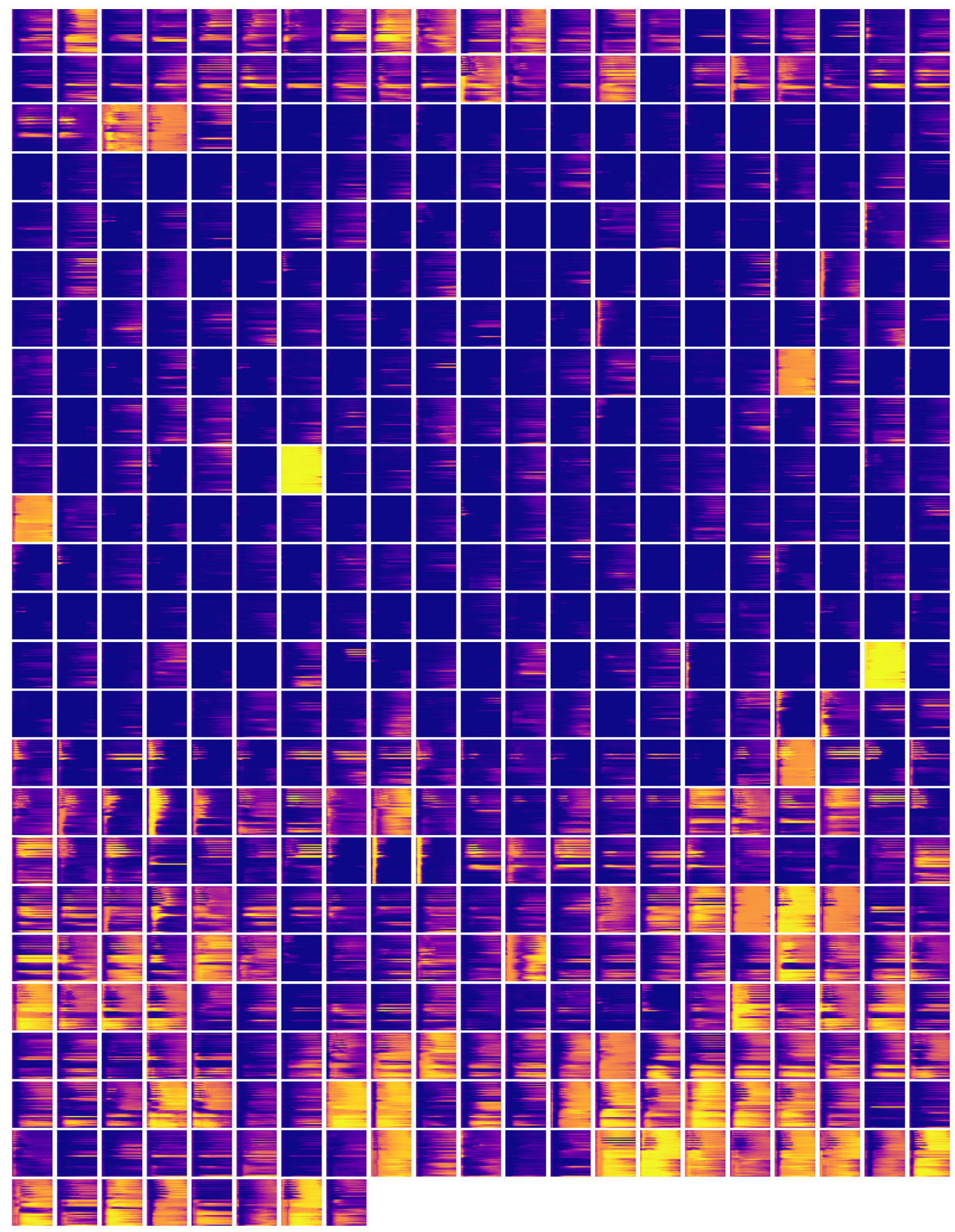

Fig. A2.2. Activations over time of all hidden units to each phoneme. Each panel displays one unit's response to each phoneme (y-axis, with the same ordering as Fig. 4 in the main article) over a period of 350ms. Units are ordered according to similarity in response profiles. 


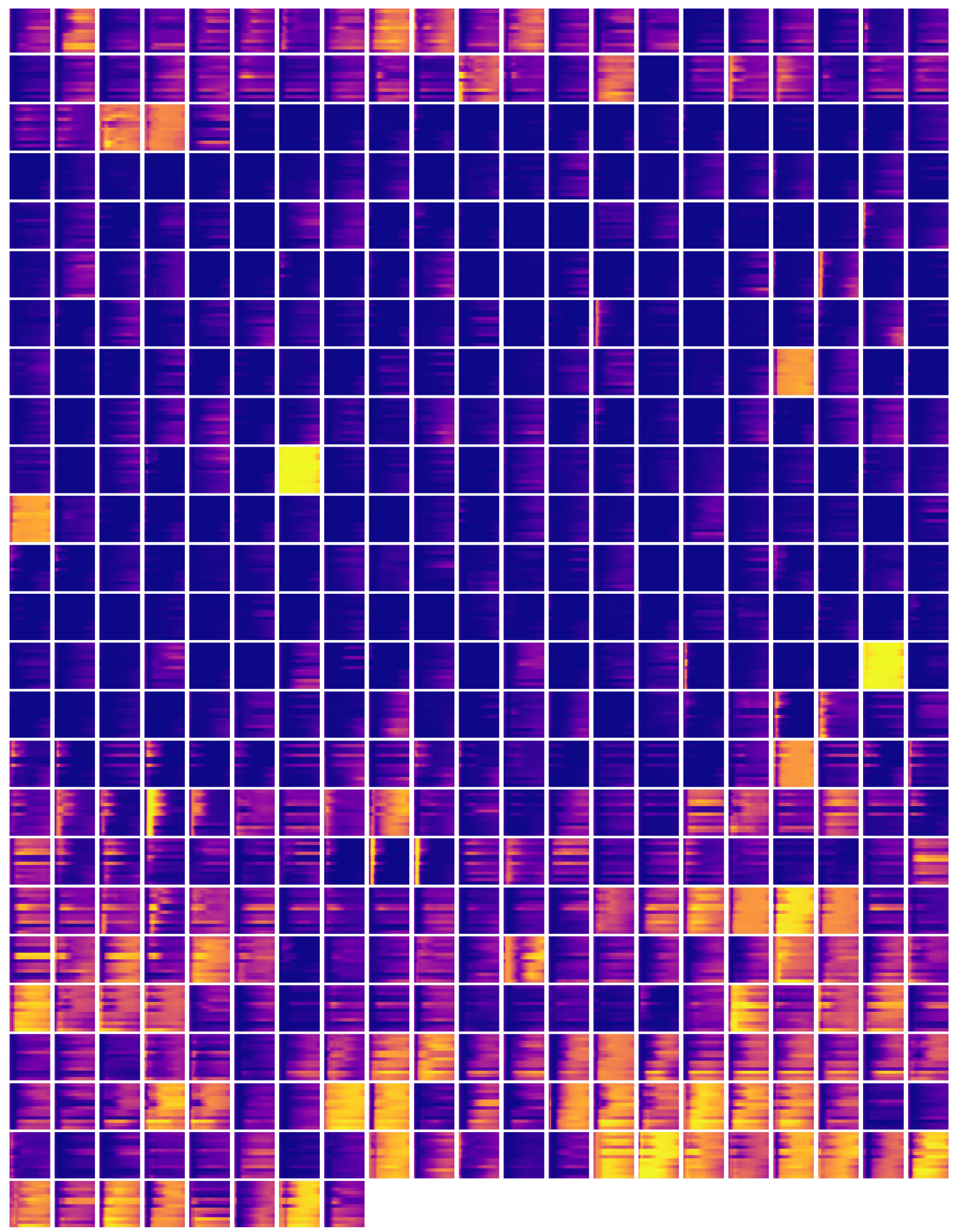

Fig. A2.3. Activations over time of all hidden units to features. Each panel displays one unit's response to each feature over a period of $350 \mathrm{~ms}$. Units are ordered according to similarity in phoneme response profiles (i.e., the same order as in Fig. S3). To see the order of features, see Fig. S5. 


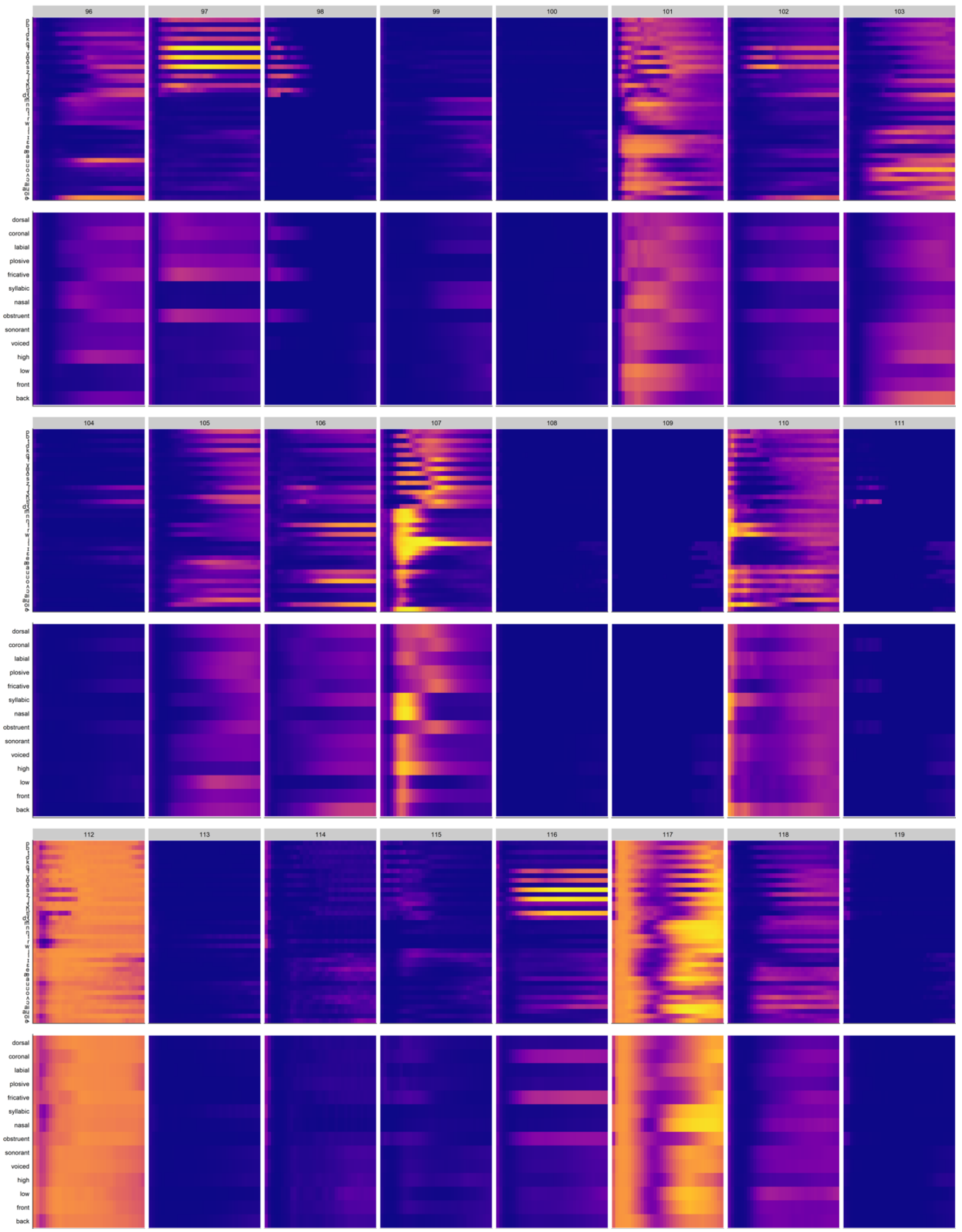

Fig. A2.4. Examples illustrating the tendency for greater sensitivity to phonemes than features. Responses of units $96-119$ to phonemes (rows 1, 3, and 5) and features (rows 2, 4, and 6). A moderate tendency for sharper, more selective responses to phonemes than features is apparent. 